\title{
Mutual Fund Theorem for continuous time markets with random coefficients
}

\author{
Nikolai Dokuchaev \\ Department of Mathematics, Trent University, Ontario, Canada
}

October 30, 2018

\begin{abstract}
We study the optimal investment problem for a continuous time incomplete market model such that the risk-free rate, the appreciation rates and the volatility of the stocks are all random; they are assumed to be independent from the driving Brownian motion, and they are supposed to be currently observable. It is shown that some weakened version of Mutual Fund Theorem holds for this market for general class of utilities; more precisely, it is shown that the supremum of expected utilities can be achieved on a sequence of strategies with a certain distribution of risky assets that does not depend on risk preferences described by different utilities.
\end{abstract}

Key words: optimal portfolio, Mutual Fund Theorem, continuous time market models.

Mathematical Subject Classification (2010): $91 \mathrm{G} 10$

\section{Introduction}

We study an optimal portfolio selection problem in a market model which consists of a risk-free bond or bank account and a finite number of risky stocks. The evolution of stock prices is described by Ito stochastic differential equations with the vector of the appreciation rates $a(t)$ and the volatility matrix $\sigma(t)$, while the bond price is exponentially increasing with a random risk free rate $r(t)$. A typical optimal portfolio selection problem is to find an investment strategy that maximizes $\mathbf{E} U(\widetilde{X}(T))$, where $\mathbf{E}$ denotes the mathematical expectation, $U(\cdot)$ is an utility function, $X(T)$ represents the wealth at final time $T$, and $\tilde{X}(T)=\exp \left(-\int_{0}^{T} r(s) d s\right) X(T)$ is the discounted wealth. There are many works devoted to different modifications of this problem (see, e.g., Merton (1969) and review in Hakansson (1997) and Karatzas and Shreve (1998)). 
Dynamic portfolio selection problems are usually studied in the framework of stochastic control. To suggest a strategy, one needs to forecast future market scenarios (or the probability distributions, or the future distributions of $r(t), a(t)$ and $\sigma(t))$. Unfortunately, the nature of financial markets is such that the choice of a hypothesis about the future distributions is always difficult to justify. In fact, it is still an open question if there is any useful information in the past prices that helps to predict the future. Respectively, there are serious reservations toward usual tools of stochastic control such as Dynamic Programming or Stochastic Maximum Principle that require knowledge of future $r(t), a(t)$ and $\sigma(t)$. It is why some special methods were developed for the financial models to deal with limited predictability.

One of this tools is the so-called Mutual Fund Theorem that says that if the distribution of the risky assets in the optimal portfolio does not depend on the investor's risk preferences (or utility function). This means that all rational investors may achieve optimality using the same mutual fund plus a saving account. Clearly, calculation of the optimal portfolio is easier in this case.

If Mutual Fund Theorem holds, then, for a typical model, portfolio stays on the efficient frontier even if there are errors in the forecast, i.e., it is optimal for some other risk preferences. This reduces the impact of forecast errors. This is another reason why it is important to know when Mutual Fund Theorem holds.

Mutual Fund Theorem was established first for the single period mean variance portfolio selection problem, i.e., for the problem with quadratic criterions. This result was a cornerstone of the modern portfolio theory. In particular, the Capital Assets Pricing Model (CAPM) is based on it. For the multi-period discrete time setting, some versions of Mutua Fund Theorem were obtained so far for problems with quadratic criterions only ( $\mathrm{Li}$ and $\mathrm{Mg}$ (1999), Dokuchaev (2010)). For the continuous time setting, Mutual Fund Theorem was obtained for portfolio selection problems with quadratic criterions as well as for more general utilities. In particular, Merton's optimal strategies for $U(x)=\delta^{-1} x^{\delta}$ and $U(x)=\log (x)$ are such that Mutual Fund Theorem holds for the case of random coefficients independent from the driving Brownian motion (Karatzas and Shreve (1998)). It is also known that Mutual Fund Theorem does not hold for power utilities in the presence of correlations (see, e.g., Brennan (1998), Feldman (2007). Khanna and Kulldorff (1999) proved that Mutual Fund Theorem theorem holds for a general utility function $U(x)$ for the case of non-random coefficient, and for a setting with consumption. Lim (2004) and Lim and Zhou (2002) found some cases Mutual Fund Theorem theorem holds for problems with quadratic criterions. Dokuchaev and Haussmann (2001) found that Mutual Theorem holds if the scalar value $\int_{0}^{T}|\theta(t)|^{2} d t$ is non-random, 
where $\theta(t)$ is the market price of risk process. Schachermayer et al (2009) found sufficient conditions for Mutual Fund Theorem expressed via replicability of the European type claims $F(Z(T))$, where $F(\cdot)$ is a deterministic function and $Z(t)$ is the discounted wealth generated by the log-optimal optimal discounted wealth process. The required replicability has to be achieved by trading of the log-optimal mutual fund with discounted wealth $Z(t)$.

It can be summarized that Mutual Fund Theorem was established so far for the following continuous time optimal portfolio selection problems:

(i) For $U(x) \equiv \log (x)$ for the case of general random coefficients $(r, a, \sigma)$;

(ii) For $U(x)=\delta^{-1} x^{\delta}, \delta \neq 0$ for the random coefficients $(r, a, \sigma)$ being independent from the driving Brownian motions;

(iii) For problems with quadratic criterions;

(iv) For general utility and for non-random coefficients $(r, a, \sigma)$;

(v) For general utility when the integral $\int_{0}^{T}|\theta(t)|^{2} d t$ is non-random;

(vi) For general utility when the claims $F(Z(T))$ can be replicated via trading of a mutual fund with the discounted wealth $Z(t)$, the deterministic functions $F$.

In fact, conditions (iv) or (v) are more restrictive than (vi).

Extension of Mutual Fund Theorem on problems (i)-(vi) was not trivial; it required significant efforts and variety of mathematical methods.

In this paper, we present one more case when Mutual Fund Theorem holds. More precisely, we found that it holds for general utility when the parameters $r(t), a(t)$ and $\sigma(t)$ are all random, they are independent from the driving Brownian motion, and they are currently observable. It is an incomplete market; it is a case of "totally unhedgeable" coefficients, according to terms from Karatzas and Shreve (1998), Chapter 6. In fact, we found that only a weakened version of Mutual Fund Theorem holds: the supremum of expected utilities can be achieved on a sequence of strategies with a certain distribution of risky assets that does not depend on utility.

\section{Definitions}

We are given a standard probability space $(\Omega, \mathcal{F}, \mathbf{P})$, where $\Omega=\{\omega\}$ is a set of elementary events, $\mathcal{F}$ is a complete $\sigma$-algebra of events, and $\mathbf{P}$ is a probability measure that describes a prior probability distributions. 


\section{Market model}

We consider a market model in a generalized Black-Scholes framework. We assume that the market consists of a risk free asset or bank account with price $B(t), t \geq 0$, and $n$ risky stocks with prices $S_{i}(t), t \geq 0, i=1,2, \ldots, n$, where $n<+\infty$ is given.

We assume that

$$
B(t)=B(0) \exp \left(\int_{0}^{t} r(s) d s\right)
$$

where $r(t)$ is the random process of the risk-free interest rate (or the short rate). We assume that $B(0)=1$. The process $B(t)$ will be used as numeraire.

The prices of the stocks evolve according to

$$
d S_{i}(t)=S_{i}(t)\left(a_{i}(t) d t+\sum_{j=1}^{n} \sigma_{i j}(t) d w_{j}(t)\right), \quad t>0,
$$

where $w(\cdot)=\left(w_{1}(\cdot), \ldots, w_{n}(\cdot)\right)$ is a standard Wiener process with independent components, $a_{i}(t)$ are the appreciation rates, and $\sigma_{i j}(t)$ are the volatility coefficients. The initial price $S_{i}(0)>0$ is a given non-random constant.

We assume that $r(t), a(t) \triangleq\left\{a_{i}(t)\right\}_{i=1}^{n}$, and $\sigma(t) \triangleq\left\{\sigma_{i j}(t)\right\}_{i, j=1}^{n}$ are currently observable uniformly bounded, measurable random processes In addition, we assume that the inverse matrix $\sigma(t)^{-1}$ is defined and bounded and $r(t) \geq 0$.

Let $\mathcal{F}_{t}$ be the filtration generated by all observable data. In particular, we assume that the processes $(S(t), r(t), a(t), \sigma(t))$ is adapted to $\mathcal{F}_{t}$, where $S(t) \triangleq\left(S_{1}(t), \ldots, S_{n}(t)\right)^{\top}$.

Set $\mu(t) \triangleq(r(t), \widetilde{a}(t), \sigma(t))$, where $\widetilde{a}(t) \triangleq a(t)-r(t) \mathbf{1}$ and $\mathbf{1} \triangleq(1,1, \ldots, 1)^{\top} \in \mathbf{R}^{n}$. The process $\mu$ represents the vector of current market parameters.

We assume that the process $\mu(t)$ is independent from $w(\cdot)$.

Let

$$
\widetilde{S}(t)=\left(\widetilde{S}_{1}(t), \ldots, \widetilde{S}_{n}(t)\right)^{\top} \triangleq \exp \left(-\int_{0}^{t} r(s) d s\right) S(t)
$$

\section{Wealth and strategies}

Let $X_{0}>0$ be the initial wealth at time $t=0$, and let $X(t)$ be the wealth at time $t>0$, $X(0)=X_{0}$. Let the process $\pi_{0}(t)$ represents the proportion of the wealth invested in the bond, $\pi_{i}(t)$ is the proportion of the wealth invested in the $i$ th stock. In other words, the process $\pi_{0}(t) X(t)$ represents the proportion of the wealth invested in the bond, $\pi_{i}(t) X(t)$ is the proportion of the wealth invested in the $i$ th stock, $\pi(t)=\left(\pi_{1}(t), \ldots, \pi_{n}(t)\right)^{\top}, t \geq 0$. We 
assume that

$$
\pi_{0}(t)+\sum_{i=1}^{n} \pi_{i}(t)=1,
$$

The case of negative $\pi_{i}$ is not excluded.

The process $\tilde{X}(t) \triangleq \exp \left(-\int_{0}^{t} r(s) d s\right) X(t)$ is called the discounted wealth.

Let $\mathbf{S}(t) \triangleq \operatorname{diag}\left(S_{1}(t), \ldots, S_{n}(t)\right)$ and $\widetilde{\mathbf{S}(\mathbf{t})} \triangleq \operatorname{diag}\left(\widetilde{S}_{1}(t), \ldots, \widetilde{S}_{n}(t)\right)$ be the diagonal matrices with the corresponding diagonal elements.

The portfolio is said to be self-financing, if

$$
d X(t)=X(t)\left(\pi(t)^{\top} \mathbf{S}(t)^{-1} d S(t)+\pi_{0}(t) B(t)^{-1} d B(t)\right)
$$

It follows that for such portfolios

$$
d \widetilde{X}(t)=\widetilde{X}(t) \pi(t)^{\top} \widetilde{\mathbf{S}}(t)^{-1} d \widetilde{S}(t)
$$

so $\pi$ alone suffices to specify the portfolio.

Let

$$
\theta(t) \triangleq \sigma(t)^{-1} \widetilde{a}(t)
$$

be the risk premium process.

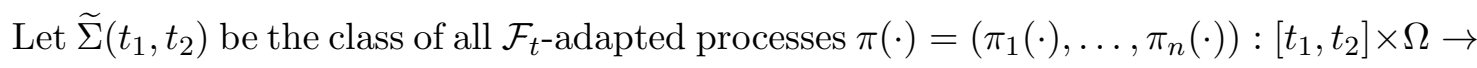
$\mathbf{R}^{n}$ such that $\sup _{t, \omega}|\pi(t, \omega)|<+\infty$ and that if $\theta(t)=0$ then $\pi(t)=0$.

We shall consider classes $\widetilde{\Sigma}\left(t_{1}, t_{2}\right)$ as classes of admissible strategies. For these strategies, $X(t)>0$ a.e..

\section{The main result}

Let $T>0$ and $X_{0}>0$ be given. Let $U(\cdot):(0,+\infty) \rightarrow \mathbf{R}$ be a given non-decreasing on $(0,+\infty)$ function.

Let

$$
J(\pi) \triangleq \mathbf{E} U\left(X\left(T, 0, X_{0}, \pi\right)\right)
$$

We will study the problem

$$
\text { Maximize } \quad J(\pi) \quad \text { over } \quad \pi(\cdot) \in \Sigma(0, T)
$$

Let $\Sigma_{M F T}\left(t_{1}, t_{2}\right)$ be the set of all strategies $\pi \in \Sigma\left(t_{1}, t_{2}\right)$ such that $\pi(t)^{\top}=$ $\nu(t) \theta(t)^{\top} \sigma(t)^{-1}$, where $\nu(t)$ is an one dimensional process adapted to $\mathcal{F}_{t}$. 
Theorem 3.1 Let the function $U$ has the form

$$
U(x)=U_{0}(x)-\sum_{k=1}^{N} U_{k}(x) x^{-\delta_{k}}+U_{N+1}(x) \log x,
$$

where $N \geq 0$ is an integer, $\delta_{k} \in(0,+\infty), k=1, \ldots, N$, and where continuous functions $U_{k}:(0,+\infty) \rightarrow \mathbf{R}$ are such that $U_{k}(x) \geq 0, k=1, \ldots, N+1$,

$$
\begin{aligned}
& \inf _{x>0} U_{0}(x)>-\infty, \\
& \sup _{x>0} U_{k}(x)<+\infty, \quad k=1, \ldots, N, \quad \sup _{x \in(0,1)} U_{N+1}(x)<+\infty .
\end{aligned}
$$

Then Mutual Fund Theorem holds in the following sense:

$$
\sup _{\pi \in \Sigma(0, T)} J(\pi)=\sup _{\pi \in \Sigma_{M F T}(0, T)} J(\pi) .
$$

Moreover, there exits a constant $C>0$ that depends only on $n$ and $\sigma(\cdot)$ such that for any $\pi \in \Sigma(0, T)$ and any $\delta>0$ there exists a strategy $\widehat{\pi} \in \Sigma_{M F T}(0, T)$ such that

$$
\begin{aligned}
& J(\widehat{\pi}) \geq J(\pi)-\delta, \\
& \sup _{t, \omega}|\widehat{\pi}(t, \omega)| \leq C \sup _{t, \omega}|\pi(t, \omega)| .
\end{aligned}
$$

Note the class of admissible $U$ is quite wide, with some restrictions on the order of singularity for utility at $x=0$ in condition (3.2).

\section{Proofs}

Note that (3.2) is not required in Lemmas 4.1 and 4.2 ,

Lemma 4.1 Let $\mu(t)=(r(t), \widetilde{a}(t), \sigma(t)$ be a non-random process and let the function $U$ be non-decreasing and continuous on $(0,+\infty)$. Then Mutual Fund Theorem holds in the following sense: for any $\pi \in \Sigma(0, T)$ and any $\delta>0$, there exists a strategy $\widehat{\pi} \in \Sigma_{M F T}(0, T)$ such that (3.5)-(3.6) hold and

$$
\widehat{\pi}(t, \omega)^{\top}=\nu(t, \omega) \theta(t)^{\top} \sigma(t)^{-1}, \quad \text { where } \quad \nu(t, \omega)=\frac{\left|\xi(t, \omega) \sigma(t)^{\top}\right|}{|\theta(t)|},
$$

if $\theta(t) \neq 0$, where $\xi(t, \omega)$ is a random $n$-dimensional $\mathcal{F}_{t}$-adapted process such that $|\xi(t, \omega)| \leq$ $\sup _{t, \omega}|\pi(t, \omega)|$. The constant $C>0$ in (3.6) depends only on $n$ and $\sigma(\cdot)$. 
Proof of Lemma 4.1. Let $\pi \in \Sigma(0, T)$ and $\delta>0$ be given. Let $C \triangleq \sup _{t, \omega}|\pi(t, \omega)|$. By the assumptions about $\Sigma(0, T)$, we have that $C<+\infty$. Let $\Sigma_{C}$ be the set of all strategies from $\tilde{\pi} \in \Sigma(0, T)$ such that $\sup _{t, \omega}|\tilde{\pi}(t, \omega)| \leq C$.

Consider the optimal control problem with the controlled process $Y(t) \triangleq \log \tilde{X}(t)$ and with admissible strategies from $\Sigma_{C}$. By Theorem V.2.5 from Krylov (1980), p.225, we obtain that there exists a so-called Markov strategy $\pi_{M}(t)=F\left(Y_{M}(t), t\right) \in \Sigma_{C}$, where $F: \mathbf{R} \times \mathbf{R} \rightarrow$ $\mathbf{R}^{n}$ is a measurable function such that the closed equation for $Y_{M}(t) \triangleq \log \widetilde{X}\left(t, 0, X_{0}, \pi_{M}\right)$ is a diffusion process and that $J\left(\widehat{\pi}_{M}\right) \geq J(\pi)-\delta$.

Further, let us apply the idea of the proof of Theorem 1 from Khanna and Kulldorff (1999) adjusted to our case of the model without consumption. Let us select $\widehat{\pi}(t)=\widehat{F}\left(Y_{M}(t), t\right) \in$ $\Sigma_{M F T}(0, T)$ such that $\widehat{\pi}(t)=f\left(Y_{M}, t\right)$, where the function $f(x, t): \mathbf{R}^{2} \rightarrow \mathbf{R}$ is defined as a solution of the finite dimensional maximization problem

$$
\text { Maximize } \quad f^{\top} \widetilde{a}(t) \quad \text { over } \quad\left\{f \in \mathbf{R}^{n}: \quad\left|f^{\top} \sigma(t)\right|=\left|F_{M}(x, t)^{\top} \sigma(t)\right|\right\}
$$

If $\theta(t) \neq 0$ than $\theta(t) \sigma(t)^{-1}$, then the solution $f=f(x, t)$ is

$$
f^{\top}=f(x, t)^{\top}=\theta(t)^{\top} \sigma(t)^{-1} \nu(x, t), \quad \text { where } \quad \nu(x, t) \triangleq \frac{\left|F_{M}(x, t)^{\top} \sigma(t)\right|}{|\theta(t)|} .
$$

If $\theta(t)=0$ then, by the choice of $\Sigma(0, T)$, we have that $\mid F_{M}(x, t)=0$, and the optimal vector is $f(x, t)=0$.

We have that

$$
\begin{aligned}
& \widetilde{X}\left(t, 0, X_{0}, \pi_{M}\right)=X_{0}+\int_{0}^{t} \widetilde{X}\left(t, 0, X_{0}, \pi_{M}\right) \pi_{M}(s)^{\top} \widetilde{\mathbf{S}}(s)^{-1} d \widetilde{S}(s) \\
& =X_{0} \exp \left(\int_{0}^{t}\left(\pi_{M}(s)^{\top} \widetilde{a}(s)-\frac{1}{2}\left|\pi_{M}(s)^{\top} \sigma(s)\right|^{2}\right) d s+\pi_{M}(s)^{\top} \sigma(s) d w(s)\right) .
\end{aligned}
$$

Hence

$$
\begin{aligned}
Y_{M}(t)=\log X_{0}+\int_{0}^{t}\left(F_{M}\left(Y_{M}(s), s\right)^{\top} \widetilde{a}(s)\right. & \left.-\frac{1}{2}\left|F_{M}\left(Y_{M}(s), s\right)^{\top} \sigma(s)\right|^{2}\right) d s \\
& +\int_{0}^{t} F_{M}\left(Y_{M}(s), s\right)^{\top} \sigma(s) d w(s) .
\end{aligned}
$$

Let $\widehat{Y}(t) \triangleq \log \widetilde{X}\left(t, 0, X_{0}, \widehat{\pi}\right)$. We have

$$
\begin{aligned}
\widehat{Y}(t)=\log X_{0}+\int_{0}^{t}\left(f\left(Y_{M}(s), s\right)^{\top} \widetilde{a}(s)\right. & \left.-\frac{1}{2}\left|f\left(Y_{M}(s), s\right)^{\top} \sigma(s)\right|^{2}\right) d s \\
+ & \int_{0}^{t} f\left(Y_{M}(s), s\right)^{\top} \sigma(s) d w(s),
\end{aligned}
$$


Let $\xi(t) \triangleq \widehat{\pi}(t)^{\top} \theta(t)-\pi_{M}(t)^{\top} \widetilde{a}(t)$. By the choice of $\widehat{\pi}$ and $f$, we have that $\xi(t) \geq 0$. Hence

$$
\begin{aligned}
\tilde{Y}(t)=\log X_{0}+\int_{0}^{t}\left(f\left(Y_{M}(s), s\right)^{\top} \widetilde{a}(s)+\xi(t)\right. & \left.-\frac{1}{2}\left|f\left(Y_{M}(s), s\right)^{\top} \sigma(s)\right|^{2}\right) d s \\
& +\int_{0}^{t} f\left(Y_{M}(s), s\right)^{\top} \sigma(s) d w(s) .
\end{aligned}
$$

It follows that $\widehat{Y}(t)=\bar{Y}(t)+\xi(t)$, where $\bar{Y}(t)$ has the same probability distribution as $Y_{M}(T)$, and $\xi(t) \geq 0$. It follows that $J(\widehat{\pi}) \geq J\left(\pi_{M}\right) \geq J(\pi)-\delta$.

In addition, we have

$$
\widehat{\pi}(t)^{\top}=\left|\pi_{M}(t)^{\top} \sigma(t)\right| e(t)^{\top} \sigma(t)^{-1}, \quad e(t) \triangleq \frac{\theta(t)}{|\theta(t)|}, \quad \theta(t) \neq 0 .
$$

Since $|e(t)|=1$ and the matrix $\sigma(t)^{-1}$ is bounded, the estimate (3.6) holds. This completes the proof of Lemma 4.1, $\square$.

Let us consider now the case when the parameters are predicable on a some given finite horizon.

Lemma 4.2 Let $U$ be non-decreasing and continuous on $(0,+\infty)$, and let there exists a finite set $\left\{t_{k}\right\}_{k=0}^{N}$ such that $0=t_{0}<t_{1}<\ldots<t_{N}=T$ and such that the values $\left.\mu(t)\right|_{t \in\left[t_{k}, t_{k+1}\right)}$ can be predicted at times $t_{k}$, meaning that $\mu(t)$ is $\mathcal{F}_{t_{k}}$-measurable for $t \in\left[t_{k}, t_{k+1}\right), k<N$. Then Mutual Fund Theorem holds in the following sense: for any $\pi \in \Sigma(0, T)$ and any $\delta>0$, there exists a strategy $\widehat{\pi} \in \Sigma_{M F T}(0, T)$ such that (3.5)-(3.6) hold and

$$
\widehat{\pi}(t, \omega)^{\top}=\nu(t, \omega) \theta(t, \omega)^{\top} \sigma(t)^{-1}, \quad \text { where } \nu(t)=\frac{\left|\xi(t, \omega) \sigma(t, \omega)^{\top}\right|}{|\theta(t, \omega)|},
$$

if $\theta(t, \omega) \neq 0$, where $\xi(t, \omega)=\xi(t, \omega)$ is a random $n$-dimensional $\mathcal{F}_{t}$-adapted process such that $|\xi(t, \omega)| \leq \sup _{t, \omega}|\pi(t, \omega)|$. The constant $C>0$ in (3.6) depends only on $n$ and $\sigma(\cdot)$.

Corollary 4.1 Lemma 4.2 holds if the conditions on $\mu$ are replaced by the following condition: there exists $\varepsilon>0$ such that $\mu(t)=(r(t), \widetilde{a}(t), \sigma(t))$ is predictable with time horizon $\varepsilon$, meaning that $\mu(t+\tau)$ is $\mathcal{F}_{t}$-measurable for any $\tau \leq \varepsilon$. Then Lemma 4.2 holds, i.e, the Mutual Fund Theorem holds in the sense of Lemma 4.2.

Proof of Lemma 4.2. Let us continue the proof of Lemma 4.2, It suffices to prove that, for any $\delta>0$ and strategy $\pi \in \Sigma(0, T)$ there exists a strategy $\widehat{\pi} \in \Sigma_{M F T}(0, T)$ such that (4.3) holds.

Clearly, it suffices to prove that, for all $z \in(0,+\infty)$, for any $\delta>0$, any $m \in\{0,1, \ldots, N-$ $1\}$, and any $\pi=\pi(z) \in \Sigma_{\varepsilon}\left(t_{m}, T\right)$, there exists $\widehat{\pi}=\widehat{\pi}(z) \in \Sigma_{\varepsilon, M F T}\left(t_{m}, T\right)$ such that

$$
\mathbf{E}\left\{U\left(\widetilde{X}\left(T, t_{m}, z, \pi\right)\right) \mid \mathcal{F}_{t_{m}}\right\} \leq \mathbf{E}\left\{U\left(\widetilde{X}\left(T, t_{m}, z, \widehat{\pi}\right)\right) \mid \mathcal{F}_{t_{m}}\right\}+\frac{N-m}{N} \delta
$$


We will use mathematical induction with decreasing $m$. First, the statement of lemma holds for $m=N-1$ by Lemma 4.1 applied on the conditional probability space. It suffices to prove that if the statement of Lemma holds for some $m+1 \leq N$ then it implies that the statement of lemma holds for $m$.

Let $z \in(0,+\infty)$ be given, and $\pi=\pi(z) \in \Sigma_{\varepsilon}\left(t_{m}, T\right)$ be a strategy.

Let $V_{N}(x)=U(x)$. For $x \in \mathbf{R}$, for $k=N-1, N-2, \ldots$, consider a sequence of functions $\widehat{\pi}_{k}: \mathbf{R} \times\left[t_{k}, t_{k+1}\right] \times \Omega \rightarrow \mathbf{R}^{n}$ and $V_{k}(x, \omega): \mathbf{R} \times \Omega \rightarrow \mathbf{R}$ such that $\widehat{\pi}_{k}(x, \cdot) \in \Sigma_{M F T}\left(t_{k}, t_{k+1}\right)$ for any $x$ and such that

$$
\begin{aligned}
& \mathbf{E}\left\{V_{k+1}\left(\tilde{X}\left(t_{k+1}, t_{k}, x, \widehat{\pi}_{k}(x, \cdot)\right)\right) \mid \mathcal{F}_{t_{k}}\right\} \\
& \quad \geq \mathbf{E}\left\{V_{k+1}\left(\tilde{X}\left(t_{k+1}, t_{k}, x, \pi\right)\right) \mid \mathcal{F}_{t_{k}}, \tilde{X}\left(t_{k}, t_{m}, z, \pi\right)=x\right\}-\frac{\delta}{N} \quad \text { a.s, } \\
& V_{k}(x) \triangleq \mathbf{E}\left\{U\left(\widetilde{X}\left(T, t_{k}, x, \tilde{\pi}_{k}(x, \cdot)\right) \mid \mathcal{F}_{t_{k}}\right\},\right.
\end{aligned}
$$

where $\tilde{\pi}_{k}(x, \cdot) \in \Sigma_{M F T}\left(t_{k}, T\right)$ is such that

$$
\begin{array}{r}
\tilde{\pi}_{k+l}(x, t)=\widehat{\pi}_{k+l}\left(\widehat{X}\left(t_{k+l}, t_{k}, x, \tilde{\pi}\right), t\right), \quad \sup _{x, t, \omega}\left|\sigma(t, \omega)^{\top} \widehat{\pi}(x, t, \omega)\right| \leq \sup _{x, t, \omega, \xi}\left|\sigma(t, \omega)^{\top} \xi\right|, \\
t \in\left[t_{k+l}, t_{k+l+1}\right], \quad l=0,1, . ., N-k-1 .
\end{array}
$$

Here supremums are taken over $x>0, t \in\left[t_{k}, t_{k+1}\right], \omega \in \Omega$, and over $\xi \in \mathbf{R}^{n}$ such that $|\xi| \leq \sup _{t, \omega}|\pi(t, \omega)|$.

These functions can be constructed recursively for $k=N-1, N-2, \ldots, m$.

Existence of $\pi_{k}$ for every steps follows from Lemma 4.1 applied on the corresponding conditional probability space.

Consider the strategy

$$
\widehat{\pi}=\widehat{\pi}(z, \cdot) \quad \text { such that } \widehat{\pi}(t)=\widetilde{\pi}_{k}(x, t)=\widehat{\pi}_{k}\left(\widehat{X}\left(t_{k}, t_{m}, x, \widehat{\pi}\right), t\right) \text { for }\left[t_{k}, t_{k+1}\right] \text {. }
$$

Let $\Pi(t)=\Pi\left(t, t_{m}, z\right) \triangleq \pi(t) \widetilde{X}\left(t, t_{m}, z, \pi\right)$. We have that, for any strategy $\pi$,

$$
\begin{aligned}
\widetilde{X}\left(T, t_{m}, z, \pi\right) & =z+\int_{t_{m}}^{T} \Pi(t)^{\top} \widetilde{\mathbf{S}}(t)^{-1} d \widetilde{S}(t) \\
& =z+\int_{t_{m}}^{T} \Pi(t)^{\top}(\widetilde{a}(t) d t+\sigma(t) d w(t)) .
\end{aligned}
$$

Let $\left.\pi_{m} \triangleq \pi\right|_{\left[t_{m}, t_{m+1}\right]}$. It follows that

$$
\begin{array}{r}
\tilde{X}\left(T, t_{m}, z, \pi\right)=\xi_{m+1}\left(\pi_{m}, z\right)+\int_{t_{m+1}}^{T} \Pi(t)^{\top}(\widetilde{a}(t) d t+\sigma(t) d w(t)) \\
=\widetilde{X}\left(T, t_{m+1}, \xi_{m+1}\left(\pi_{m}, z\right), \pi\right),
\end{array}
$$


where

$$
\xi_{m+1}\left(\pi_{m}, z\right) \triangleq \widetilde{X}\left(t_{m+1}, t_{m}, z, \pi\right)=z+\int_{t_{m}}^{t_{m+1}} \Pi(t)(\widetilde{a}(t) d t+\sigma(t) d w(t)) .
$$

Further,

$$
\begin{array}{r}
\mathbf{E}\left\{U\left(\widetilde{X}\left(T, t_{m}, z, \pi\right)\right) \mid \mathcal{F}_{t_{m}}\right\}=\mathbf{E}\left\{\mathbf{E}\left\{U\left(\widetilde{X}\left(T, t_{m}, z, \pi\right)\right) \mid \mathcal{F}_{t_{m+1}}\right\} \mid \mathcal{F}_{t_{m}}\right\} \\
=\mathbf{E}\left\{\mathbf{E}\left\{U\left(\xi_{m+1}\left(\pi_{m}, z\right)+\int_{t_{m+1}}^{T} \Pi(t)(\widetilde{a}(t) d t+\sigma(t) d w(t))\right) \mid \mathcal{F}_{t_{m+1}}\right\} \mid \mathcal{F}_{t_{m}}\right\} .
\end{array}
$$

The equalities and inequalities here holds a.s., as well as inequalities and equalities for conditional expectations below.

By the definitions and by the induction assumption that (4.2) holds with $m$ replaced by $m+1$, we obtain that

$$
\begin{aligned}
& \mathbf{E}\left\{U\left(\widetilde{X}\left(T, t_{m+1}, \xi_{m+1}\left(\pi_{m}, z\right), \pi\right)\right) \mid \mathcal{F}_{t_{m+1}}\right\} \\
& =\mathbf{E}\left\{U\left(\xi_{m+1}\left(\pi_{m}, z\right)+\int_{t_{m+1}}^{T} \Pi(t)^{\top}(\widetilde{a}(t) d t+\sigma(t) d w(t))\right) \mid \mathcal{F}_{t_{m+1}}\right\} \\
& \leq V_{m+1}\left(\xi_{m+1}\left(\pi_{m}, z\right)\right)+\frac{N-m-1}{N} \delta
\end{aligned}
$$

Hence

$$
\mathbf{E}\left\{U\left(\widetilde{X}\left(T, t_{m}, z, \pi\right)\right) \mid \mathcal{F}_{t_{m}}\right\} \leq \mathbf{E}\left\{V_{m+1}\left(\xi_{m+1}\left(\pi_{m}, z\right)\right) \mid \mathcal{F}_{t_{m}}\right\}+\frac{N-m-1}{N} \delta .
$$

Further, by the choice of $\widehat{\pi}_{m}$, we obtain that

$$
\begin{aligned}
& \mathbf{E}\left\{V_{m+1}\left(\xi_{m+1}\left(\pi_{m}, z\right)\right) \mid \mathcal{F}_{t_{m}}\right\}=\mathbf{E}\left\{V_{m+1}\left(\widetilde{X}\left(t_{m+1}, t_{m}, z, \pi\right)\right) \mid \mathcal{F}_{t_{m}}\right\} \\
& \leq \mathbf{E}\left\{V_{m+1}\left(\widetilde{X}\left(t_{m+1}, t_{m}, z, \widehat{\pi}\right)\right) \mid \mathcal{F}_{t_{m}}\right\}+\frac{\delta}{N} \\
& =\mathbf{E}\left\{V_{m+1}\left(\xi_{m+1}\left(\widehat{\pi}_{m}, z\right)\right) \mid \mathcal{F}_{t_{m}}\right\}+\frac{\delta}{N}
\end{aligned}
$$

By the definitions,

$$
\begin{aligned}
V_{m+1}\left(\xi_{m+1}\left(\widehat{\pi}_{m}, z\right)\right) & =V_{m+1}\left(\widetilde{X}\left(t_{m+1}, t_{m}, z, \widehat{\pi}\right), z\right) \\
& =\mathbf{E}\left\{U\left(\widetilde{X}\left(T, t_{m+1}, \widetilde{X}\left(t_{m+1}, t_{m}, z, \widehat{\pi}\right), \widehat{\pi}\right)\right) \mid \mathcal{F}_{t_{m+1}}\right\}
\end{aligned}
$$

By the version of the Markov property described in Theorem II.9.4 from Krylov (1980) and applied on the conditional space given $\mathcal{F}_{t_{m}}$, we have that the right hand part of equality (4.5) can be rewritten as

$$
\mathbf{E}\left\{V_{m+1}\left(\xi_{m+1}\left(\widehat{\pi}_{m}, z\right)\right) \mid \mathcal{F}_{t_{m}}\right\}=\mathbf{E}\left\{U\left(\widetilde{X}\left(T, t_{m}, z, \widehat{\pi}\right) \mid \mathcal{F}_{t_{m}}\right\}\right.
$$


We used here that $\mu_{\varepsilon}(\cdot)$ is independent from $w(\cdot)$. By (4.3)-(4.6), it follows that

$$
\mathbf{E}\left\{U\left(\widetilde{X}\left(T, t_{m}, z, \pi\right)\right) \mid \mathcal{F}_{t_{m}}\right\} \leq \mathbf{E}\left\{U\left(\widetilde{X}\left(T, t_{m}, z, \widehat{\pi}\right)\right) \mid \mathcal{F}_{t_{m}}\right\}-\frac{N-m}{N} \delta .
$$

Since it holds for any $\pi \in \Sigma\left(t_{m}, T\right)$, it follows that Lemma 4.2 holds.

Lemma 4.3 Theorem 3.1 holds under additional condition that $\sup _{x>0} U_{k}(x)<+\infty$ in (3.2) for $k=0$ and $k=N+1$.

Proof. Let $t \wedge s=\min (t, s)$,

$$
r_{\varepsilon}(t) \triangleq \frac{1}{\varepsilon} \int_{(t-2 \varepsilon) \wedge 0}^{(t-\varepsilon) \wedge 0} r(s) d s, \quad a_{\varepsilon}(t) \triangleq \frac{1}{\varepsilon} \int_{(t-2 \varepsilon) \wedge 0}^{(t-\varepsilon) \wedge 0} a(s) d s, \quad \sigma_{\varepsilon}(t) \triangleq \frac{1}{\varepsilon} \int_{(t-2 \varepsilon) \wedge 0}^{(t-\varepsilon) \wedge 0} \sigma(s) d s,
$$

and let

$$
\mu_{\varepsilon}(t) \triangleq\left(r_{\varepsilon}(t), \widetilde{a}_{\varepsilon}(t), \sigma_{\varepsilon}(t)\right), \quad \widetilde{a}_{\varepsilon}(t) \triangleq a_{\varepsilon}(t)-r_{\varepsilon}(t), \quad \theta_{\varepsilon}(t) \triangleq \sigma_{\varepsilon}(t)^{-1} \widetilde{a}_{\varepsilon}(t) .
$$

Consider a sequence $\varepsilon=\varepsilon_{N}=1 / N \rightarrow 0, N=1,2, \ldots$. For every $\varepsilon=\varepsilon_{i}$, consider a finite sequences of times $\left\{t_{j}\right\}_{j=0}^{N}$ such that $t_{k+1}=t_{k}+\varepsilon$.

Let $\mathcal{F}_{t}^{\mu, \varepsilon}$ be the filtration generated by $\mu_{\varepsilon}(t)$ and let $\mathcal{F}_{t}^{\varepsilon}$ be the filtration generated by $\left(\mu_{\varepsilon}(t), w(t)\right)$.

Let $\widetilde{\Sigma}(0, T)$ be the class of all $\mathcal{F}_{t}^{\varepsilon}$-adapted processes $\pi(\cdot)=\left(\pi_{1}(\cdot), \ldots, \pi_{n}(\cdot)\right):[0, T] \times \Omega \rightarrow$ $\mathbf{R}^{n}$ such that $\sup _{t, \omega}|\pi(t, \omega)|<+\infty$ and that if $\theta_{\varepsilon}(t)=0$ then $\pi(t)=0$.

Further, let $\Sigma_{\varepsilon, M F T}(0, T)$ denote the set of strategies from $\Sigma_{\varepsilon}(0, T)$ that have the form $\pi(t)=\nu(t) \sigma_{\varepsilon}(t)^{-1} \theta_{\varepsilon}(t)$, where $\nu_{\varepsilon}(t)$ is an one dimensional process adapted to $\mathcal{F}_{t}^{\varepsilon}$.

For $\varepsilon>0$, let

$$
J_{\varepsilon}(\pi) \triangleq \mathbf{E} U\left(\widetilde{X}_{\varepsilon}\left(T, 0, X_{0}, \pi\right)\right),
$$

where $\tilde{X}_{\varepsilon}\left(T, 0, X_{0}, \pi\right)$ is the discounted wealth for the model with $\mu$ replaced by $\mu=\mu_{\varepsilon}$ for the strategy $\pi$ given that $\tilde{X}(0)=X_{0}$. The case of $\varepsilon=0$ corresponds to the original model; in this case, the discounted wealth is denoted as $\widetilde{X}\left(T, 0, X_{0}, \pi\right)$.

Note that the market models with $\mu=\mu_{\varepsilon}$ are such that assumptions of Lemma 4.2 are satisfied for $\varepsilon>0$.

Let $\delta>0$ be given. Let $\pi \in \Sigma(0, T)$ be such that

$$
J(\pi) \geq \inf _{\pi \in \Sigma(0, T)} J(\pi)-\frac{\delta}{4} .
$$


Let $\widetilde{X}(t)=\widetilde{X}\left(T, 0, X_{0}, \pi\right)$. By the choice of $\Sigma(0, T)$, we have that $C_{\pi} \triangleq \sup _{t, \omega}|\pi(t, \omega)|<+\infty$. Let

$$
\pi_{\varepsilon}(t) \triangleq \frac{1}{\varepsilon} \int_{(t-2 \varepsilon) \wedge 0}^{(t-\varepsilon) \wedge 0} \pi(s) d s .
$$

Clearly, $\pi_{\varepsilon} \in \Sigma_{\varepsilon}(0, T)$. By Lemma 3 from Shilov and Gurevich (1967), Chapter IV, Section 5 , it follows that

$$
\mu_{\varepsilon} \rightarrow \mu, \quad \pi_{\varepsilon} \rightarrow \pi \quad \text { as } \quad \varepsilon \rightarrow 0+\quad \text { a.e. on } \quad[0, T] \times \Omega \text {. }
$$

We have that

$$
\begin{aligned}
& \widetilde{X}\left(T, 0, X_{0}, \pi\right)=X_{0}+\int_{0}^{T} \widetilde{X}\left(t, 0, X_{0}, \pi\right) \pi(t)^{\top} \widetilde{\mathbf{S}}(t)^{-1} d \widetilde{S}(t) \\
& =X_{0} \exp \left[\int_{0}^{T} \pi(t)^{\top} \widetilde{a}(t) d t-\frac{1}{2} \int_{0}^{T}\left|\pi(t)^{\top} \sigma(t)\right|^{2} d t+\int_{0}^{T} \pi(t)^{\top} \sigma(t) d w(t)\right] .
\end{aligned}
$$

Similarly,

$$
\begin{aligned}
& \widetilde{X}_{\varepsilon}\left(T, 0, X_{0}, \pi_{\varepsilon}\right) \\
& =X_{0} \exp \left[\int_{0}^{T} \pi_{\varepsilon}(t)^{\top} \widetilde{a}_{\varepsilon}(t) d t-\frac{1}{2} \int_{0}^{T}\left|\pi_{\varepsilon}(t)^{\top} \sigma_{\varepsilon}(t)\right|^{2} d t+\int_{0}^{T} \pi_{\varepsilon}(t)^{\top} \sigma_{\varepsilon}(t) d w(t)\right] .
\end{aligned}
$$

Let $Y_{\varepsilon, \varepsilon}(t) \triangleq \log X_{\varepsilon}\left(t, 0, X_{0}, \pi_{\varepsilon}\right)$ and $Y(t) \triangleq \log X_{\varepsilon}\left(t, 0, X_{0}, \pi_{\varepsilon}\right)$.

Clearly,

$$
\mathbf{E}\left|Y_{\varepsilon, \varepsilon}(T)-Y(T)\right|^{2} \rightarrow 0 \quad \text { as } \quad \varepsilon \rightarrow 0
$$

It follows that there exists a subsequence $\left\{\varepsilon_{i}\right\}$ such that

$$
Y_{\varepsilon, \varepsilon}(T) \rightarrow Y(T) \quad \text { a.s. } \quad \text { as } \quad \varepsilon=\varepsilon_{i} \rightarrow 0
$$

By the assumptions, all functions $U_{k}$ are bounded. By Lebesgue's Dominated Convergence Theorem, this subsequence $\left\{\varepsilon_{i}\right\}$ is such that

$$
\begin{array}{r}
\mathbf{E} \mid U_{k}\left(\widetilde{X}_{\varepsilon}\left(T, 0, X_{0}, \pi\right)\right)-U_{k}\left(\left.\widetilde{X}\left(T, 0, X_{0}, \pi\right)\right|^{2} \rightarrow 0 \quad \text { as } \quad \varepsilon=\varepsilon_{i} \rightarrow 0\right. \\
k=0,1, \ldots, N+1 .
\end{array}
$$

By (4.11), it follows that

$$
\mathbf{E} U_{0}\left(\widetilde{X}_{\varepsilon}\left(T, 0, X_{0}, \pi\right)\right) \rightarrow \mathbf{E} U_{0}\left(\widetilde{X}\left(T, 0, X_{0}, \pi\right) \quad \text { as } \quad \varepsilon=\varepsilon_{i} \rightarrow 0 .\right.
$$

By (4.11) and (4.9), it follows that

$$
\mathbf{E} U_{N+1}\left(\widetilde{X}_{\varepsilon}\left(T, 0, X_{0}, \pi_{\varepsilon}\right)\right) Y_{\varepsilon, \varepsilon}(T) \rightarrow \mathbf{E} U_{N+1}\left(\widetilde{X}\left(T, 0, X_{0}, \pi\right) Y(T) \quad \text { as } \quad \varepsilon=\varepsilon_{i} \rightarrow 0\right.
$$


Further, let $k \in 1, \ldots, N$, and let

$$
z_{\varepsilon, \varepsilon}(t) \triangleq X_{\varepsilon}\left(t, 0, X_{0}, \pi_{\varepsilon}\right)^{\delta_{k}}=\exp \left(\delta_{k} Y_{\varepsilon, e}(t)\right), \quad z(t) \triangleq X\left(t, 0, X_{0}, \pi\right)^{\delta_{k}}=\exp \left(\delta_{k} Y(t)\right) .
$$

By Ito formula, we obtain

$$
d z_{\varepsilon, \varepsilon}(t)=z_{\varepsilon, \varepsilon}(t)\left(\delta_{k} d Y_{\varepsilon, \varepsilon}(t)+\frac{1}{2} \delta_{k}^{2}\left|\pi_{\varepsilon, \varepsilon}(t)^{\top} \sigma_{\varepsilon}(t)\right|^{2} d t\right), \quad z_{\varepsilon, \varepsilon}(0)=X(0)^{\delta_{k}},
$$

where

$$
d Y_{\varepsilon}(t)=\pi_{\varepsilon}(t)^{\top} \widetilde{a}_{\varepsilon}(t) d t-\frac{1}{2}\left|\pi_{\varepsilon}(t)^{\top} \sigma_{\varepsilon}(t)\right|^{2} d t+\pi_{\varepsilon}(t)^{\top} \sigma_{\varepsilon}(t) d w(t) .
$$

Similarly, we obtain

$$
d z(t)=z(t)\left(\delta_{k} d Y(t)+\frac{1}{2} \delta_{k}^{2}\left|\pi(t)^{\top} \sigma(t)\right|^{2} d t\right), \quad z(0)=X(0)^{\delta_{k}},
$$

where

$$
d Y(t)=\pi(t)^{\top} \widetilde{a}(t) d t-\frac{1}{2}\left|\pi(t)^{\top} \sigma(t)\right|^{2} d t+\pi(t)^{\top} \sigma(t) d w(t) .
$$

By Theorem II.8.1 from Krylov (1980), p.102, we have that $\mathbf{E}\left|z_{\varepsilon, \varepsilon}(T)-z(T)\right|^{2} \rightarrow 0$ as $\varepsilon=\varepsilon_{i} \rightarrow 0$ for any $k=1, \ldots, N$. By (4.11), we obtain for $k=1, \ldots, N$ that

$$
\mathbf{E} U_{k}\left(\widetilde{X}_{\varepsilon}\left(T, 0, X_{0}, \pi_{\varepsilon}\right)\right) z_{\varepsilon, \varepsilon}(T) \rightarrow \mathbf{E} U_{k}\left(\widetilde{X}\left(T, 0, X_{0}, \pi\right) z(T) \quad \text { as } \quad \varepsilon=\varepsilon_{i} \rightarrow 0 .\right.
$$

By (4.12)-(4.14), we obtain that

$$
J_{\varepsilon}\left(\pi_{\varepsilon}\right)=\mathbf{E} U\left(\widetilde{X}_{\varepsilon}\left(T, 0, X_{0}, \pi_{\varepsilon}\right)\right) \rightarrow J(\pi)=\mathbf{E} U\left(\widetilde{X}\left(T, 0, X_{0}, \pi\right) \quad \text { as } \quad \varepsilon=\varepsilon_{i} \rightarrow 0 .\right.
$$

It follows that there exists $N_{1}>0$ such that, for every $i \geq N_{1}$,

$$
J_{\varepsilon}\left(\pi_{\varepsilon}\right) \geq J(\pi)-\frac{\delta}{4}, \quad \varepsilon=\varepsilon_{i} .
$$

Let $\widehat{\pi}_{\varepsilon, \varepsilon} \in \Sigma_{\varepsilon, M F T}(0, T)$ be the strategy defined in Lemma 4.2 as a strategy that outperform the strategy $\pi_{\varepsilon}$ for the market with $\mu=\mu_{\varepsilon}$, i.e., such that $\nu_{\varepsilon}(t)$ is $\mathcal{F}_{t}^{\varepsilon}$-adapted process and

$$
J_{\varepsilon}\left(\pi_{\varepsilon, \varepsilon}\right) \geq J_{\varepsilon}\left(\pi_{\varepsilon}\right)-\frac{\delta}{4}
$$

Following the proof of Lemma 4.1 we obtain similarly to (4.1) that, if $\theta(t) \neq 0$, then

$$
\pi_{\varepsilon, \varepsilon}(t)^{\top}=\theta_{\varepsilon}(t)^{\top} \sigma_{\varepsilon}(t)^{-1} \nu_{\varepsilon}(x, t), \quad \text { where } \quad \nu_{\varepsilon}(t)=\frac{\left|\xi_{\varepsilon}(t, \omega)^{\top} \sigma_{\varepsilon}(t)\right|}{\left|\theta_{\varepsilon}(t)\right|},
$$

and where $\xi_{\varepsilon}(t, \omega)$ is a $n$-dimensional vector such that $\left|\xi_{\varepsilon}(t, \omega)\right| \leq\left|\pi_{\varepsilon}(t, \omega)\right|$. If $\theta(t)=0$ then $\widehat{\pi}_{\varepsilon, \varepsilon}(t)=0$. 
By estimate (3.6) in Lemma 4.2, we have that

$$
\sup _{t, \omega, \varepsilon}\left|\pi_{\varepsilon, \varepsilon}(t, \omega)\right| \leq C \sup _{t, \omega, \varepsilon}\left|\pi_{\varepsilon}(t, \omega)\right| \leq C \sup _{t, \omega}|\pi(t, \omega)|,
$$

where $C=C(n, \sigma)>0$ is a constant.

Let

$$
\begin{aligned}
& \pi_{\varepsilon, 0}(t)^{\top} \triangleq \frac{\left|\theta_{\varepsilon}(t)\right|}{|\theta(t)|} \nu_{\varepsilon}(t) \theta(t)^{\top} \sigma(t)^{-1} \quad \text { if } \quad \theta(t) \neq 0, \theta_{\varepsilon}(t) \neq 0, \\
& \pi_{\varepsilon, 0}(t)=0 \quad \text { if } \quad \theta(t)=0 \quad \text { or } \quad \theta_{\varepsilon}(t)=0 .
\end{aligned}
$$

It follows that, if $\theta(t) \neq 0, \theta_{\varepsilon}(t) \neq 0$

$$
\pi_{\varepsilon, 0}(t)^{\top}=\frac{\left|\theta_{\varepsilon}(t)\right|}{|\theta(t)|} \frac{\left|\xi_{\varepsilon}(t, \omega)^{\top} \sigma_{\varepsilon}(t)\right|}{\left|\theta_{\varepsilon}(t)\right|} \theta(t)^{\top} \sigma(t)^{-1}=\frac{\left|\xi_{\varepsilon}(t, \omega)^{\top} \sigma_{\varepsilon}(t)\right|}{|\theta(t)|} \theta(t)^{\top} \sigma(t)^{-1} .
$$

Hence

$$
\sup _{t, \omega, \varepsilon}\left|\pi_{\varepsilon, 0}(t, \omega)\right| \leq C \sup _{t, \omega, \varepsilon}\left|\pi_{\varepsilon}(t, \omega)\right| \leq C \sup _{t, \omega}|\pi(t, \omega)|,
$$

where $C=C(n, \sigma)$ is a constant that depends only on $n$ and $\sigma$.

The equations for $\widetilde{X}_{\varepsilon}\left(T, 0, X_{0}, \pi_{\varepsilon, \varepsilon}\right)$ and $\widetilde{X}_{\varepsilon}\left(T, 0, X_{0}, \pi_{\varepsilon, 0}\right)$ are similar to equations (4.7)(4.8). Clearly, $\pi_{\varepsilon, \varepsilon}(t, \omega)-\pi_{\varepsilon, 0}(t, \omega) \rightarrow 0$ a.e.. Using (4.17)-(4.18), we obtain that $\mathbf{E}\left|\log \tilde{X}_{\varepsilon}\left(T, 0, X_{0}, \pi_{\varepsilon, \varepsilon}\right)-\log \tilde{X}\left(T, 0, X_{0}, \pi_{\varepsilon, 0}\right)\right|^{2} \rightarrow 0$ as $\varepsilon \rightarrow 0$. It follows that there exists another subsequence $\left\{\varepsilon_{i}\right\}$ (a subsequence of the subsequence from (4.10)) such that $\varepsilon_{i} \rightarrow 0$ and $\log \widetilde{X}_{\varepsilon}\left(T, 0, X_{0}, \pi_{\varepsilon, \varepsilon}\right)-\log \tilde{X}\left(T, 0, X_{0}, \pi_{\varepsilon, 0}\right) \rightarrow 0$ a.s. as $\varepsilon=\varepsilon_{i} \rightarrow 0$. Similarly to (4.12)(4.15), we obtain that this subsequence $\left\{\varepsilon_{i}\right\}$ is such that

$$
J_{\varepsilon}\left(\pi_{\varepsilon, \varepsilon}\right)-J_{\varepsilon}\left(\pi_{\varepsilon, 0}\right)=\mathbf{E} U\left(\widetilde{X}_{\varepsilon}\left(T, 0, X_{0}, \pi_{\varepsilon, \varepsilon}\right)\right)-\mathbf{E} U\left(\tilde{X}\left(T, 0, X_{0}, \pi_{\varepsilon, 0}\right) \rightarrow 0\right.
$$

as $\varepsilon=\varepsilon_{i} \rightarrow 0$. It follows that there exists $N>N_{1}>0$ such that, for every $i \geq N$,

$$
J_{0}\left(\pi_{\varepsilon, 0}\right) \geq J_{\varepsilon}\left(\pi_{\varepsilon, \varepsilon}\right)-\frac{\delta}{4}, \quad \varepsilon=\varepsilon_{i}
$$

Finally, we obtain that

$$
J_{0}\left(\pi_{\varepsilon, 0}\right) \geq J_{\varepsilon}\left(\pi_{\varepsilon, \varepsilon}\right)-\frac{\delta}{4} \geq J_{\varepsilon}\left(\pi_{\varepsilon}\right)-\frac{\delta}{2} \geq J_{0}(\pi)-\frac{3 \delta}{4}, \quad \varepsilon=\varepsilon_{i}
$$

This completes the proof of Lemma 4.3,

Proof of Theorem 3.1. It suffices to show that there exists $C>0$ such that, for any $\delta>0$ and $\pi \in \Sigma(0, T)$, there exists $\widehat{\pi} \in \Sigma_{M F T}(0, T)$ such that (3.5)-(3.6) hold. 
For $K>0$, let $U^{(K)}(x)$ be defined by (3.2) with $U_{0}$ replaced by $\min \left(U_{0}(x), K\right)$ and with $U_{N+1}$ replaced by $\min \left(U_{N+1}(x), K\right)$. Let $\mathcal{J}_{K}(\pi)=\mathbf{E} U^{(K)}\left(\widetilde{X}\left(T, 0, X_{0}, \pi\right)\right.$.

Let $C>0$ be the constant (3.6) that exists by Lemma 4.3 for all $K>0$. (Note that this constant does not depend on $K)$. Let $\delta>0$ and $\pi \in \Sigma(0, T)$ be given, Clearly, there exists $K>0$ such that $\mathcal{J}_{K}(\pi) \geq J(\pi)-\delta / 2$. By Lemma 4.3, there exists $\widehat{\pi} \in \Sigma_{M F T}(0, T)$ such that $\mathcal{J}_{K}(\widehat{\pi}) \geq \mathcal{J}_{K}(\pi)-\delta / 2$ and (3.6) holds. In addition, we have that $\mathcal{J}_{K}(\widehat{\pi}) \geq J(\widehat{\pi})$ for large enough $K$ (it suffices to take $K>\sup _{x \in(0,1)} U_{N+1}(x)$ ). For these $K$, we have that $J(\widehat{\pi}) \geq \mathcal{J}_{K}(\pi) \geq J(\pi)-\delta$. Then the proof follows.

\section{Discussion and comments}

(i) Theorem 3.1 represents a weakened version of Mutual Fund Theorem since it states only suboptimality of the strategies from the required class. A stronger version of this theorem is known for many special cases. In particular, there are stronger versions of Lemma 4.1, see, e.g., Khanna and Kulldorff (1999), Dokuchaev and Haussmann (2001), Schachermayer et al (2009). Let us explain why these versions of Lemma 4.1 cannot be applied in our proof.

Khanna and Kulldorff (1999) proved that a strategy from a class similar to $\Sigma_{M F T}$ can outperform any Markov strategies. Our setting with random parameters requires to include strategies that are not necessary Markov.

Schachermayer et al (2009)) found that the Mutual Fund Theorem holds for a market where claims $F(Z(T))$ can be replicated via trading of a mutual fund with the discounted price $Z(t)$ for deterministic functions $F$. Here $Z(t)$ is the log-optimal discounted wealth such that

$$
d Z(t)=Z(t) \theta(t)^{\top} \sigma(t)^{-1} \mathbf{S}(t)^{-1} d S(t), \quad Z(0)=1 .
$$

In the same framework, Dokuchaev and Haussmann (2001) found that Mutual Fund

Theorem holds in a more special case, when the scalar value $\int_{0}^{T}|\theta(t)|^{2} d t$ is non-random. In this case, there is the required replicability of claims $F(Z(T))$. However, these results cannot replace Lemma 4.1, because they require certain special properties for $U$ and for the functions $V_{m}$ in the proof of Lemma 4.2. If we assume these properties for $U$, it is not clear how to prove that they will be transferred to $V_{m}$.

(ii) It can be seen from the proofs above that, in many cases of random $\mu$, the suboptimal terminal discounted wealth cannot be presented as $F(Z(T))$ for a deterministic function 
$F: \mathbf{R} \rightarrow \mathbf{R}$. Respectively, these cases are not be covered by the method based on the replication of these claims (Schachermayer et al (2009), Dokuchaev and Haussmann (2001)).

(iii) The condition (3.2) in Theorem 3.1 restricts the choice of singularity for admissible utility functions $U$ at $x=0$. However, this condition is rather technical; we need it ensure the transfer from the market model from Lemma 4.2 to the more general market model in Theorem 3.1. However, the model in Lemma 4.2 is quite reasonable itself, since it is natural to assume some stability and predictability of the parameters of the distributions; this assumption is required by any statistical analysis. There are many well developed methods that may help to forecast the market parameters on a small enough horizon $\varepsilon>0$; in particular, a frequency criterion of predictability on a finite horizon can be found in Dokuchaev (2010).

(iv) It can be noted also that the construction of suboptimal strategies from the proof above shows that, in the general case, these strategies cannot be presented as $\pi(t)=$ $f(X(t), S(t), \mu(t), t)$, where $f$ is a deterministic function. This means that dynamic programming method cannot be applied directly to this model.

(v) In our setting, we assumed that the admissible strategies are such that if $\theta(t)=0$ then $\pi(t)=0$. In fact, our version of Mutual Fund Theorem does not necessary hold for a wide class without this restriction given our class of utilities. For instance, for a convex function $U(x)=x^{2}$ and $\theta(t) \equiv 0$, the only strategy $\pi_{M F T}$ from Mutual Fund Theorem is zero; however, this strategy is outperformed by any non-trivial strategy.

\section{References}

Brennan, M.J. (1998). The role of learning in dynamic portfolio decisions. European Finance Review 1, 295-306.

Dokuchaev, N., and Haussmann, U. (2001). Optimal portfolio selection and compression in an incomplete market. Quantitative Finance, 1, 336-345.

Dokuchaev, N. (2010). Mean variance and goal achieving portfolio for discrete-time market with currently observable source of correlations. ESAIM: Control, Optimisation and Calculus of Variations, in press.

Dokuchaev, N. (2010). Predictability on finite horizon for processes with exponential 
decrease of energy on higher frequencies. Signal processing 90 Issue 2, February 2010, 696701 (in press).

Feldman, D. (2007). Incomplete Information Equilibria: Separation Theorems and Other Myths. Annals of Operations Research 151, 119-149.

Karatzas, I., and Shreve, S.E. (1998). Methods of Mathematical Finance, Springer-Verlag, New York.

Khanna A.,, Kulldorff M. (1999). A generalization of the mutual fund theorem. Finance and Stochastics 3, 167185 (1999).

Krylov, N.V. (1980). Controlled diffusion processes. Shpringer-Verlag.

Li, D., and Ng, W.L. (2000). Optimal portfolio selection: multi-period mean-variance optimization. Mathematical Finance 10 (3), 387-406.

Lim, A. (2004). Quadratic hedging and mean-variance portfolio selection with random parameters in an incomplete market. Mathematics of Operations Research 29, iss.1, 132 161.

Lim, A., and Zhou, X.Y. (2002). Mean-variance portfolio selection with random parameters in a complete market. Mathematics of Operations Research 27, iss.1, 101 - 120.

Schachermayer, W., Srbu, M., Taflin, E. (2009). In which financial markets do mutual fund theorems hold true? Finance and Stochastics 13, 49-77.

Shilov G.E, Gurevich B.L (1967). Integral, measure and derivative: a unified approach. Nauka, Moscow. 